\title{
El consumo de las redes sociales en Ecuador. Una encuesta exploratoria en Riobamba y Quito (2013 - 14)
}

\begin{abstract}
Resumen
La comunicación digital ecuatoriana se encuentra relativamente escalonada, debido particularmente a la baja tasa de conexión a Internet que hay en la República andina: según cifras oficiales, solo cuatro de cada diez ecuatorianos, en 2013, tenían conexión a la red. Asimismo, el contexto comunicacional está en un interesante proceso de transformación, a través de la aprobación de instrumentos como la Ley Orgánica de Comunicación en 2013, la cual, a pesar de la exhaustividad, no regula los contenidos comunicacionales emitidos por los usuarios en las redes sociales. En el siguiente artículo, mediante una encuesta orientada a ciudadanos de dos de las principales ciudades del país -Quito y Riobamba- planteamos determinar de forma exploratoria el consumo de las redes sociales en estos entornos urbanos, así como las posibles diferencias etarias que podrían darse. Los resultados señalan que YouTube, Facebook y Google son las principales redes sociales utilizadas por los ecuatorianos; asimismo, hemos confirmado la brecha intergeneracional e interprovincial, al establecer que la mayor parte de la atención se concentraba sobre todo en las redes sociales convencionales y en las tendencias icónicas y en esas otras que permitían un uso esencialmente interpersonal.
\end{abstract}

\section{Palabras clave}

Redes sociales; Ecuador; Ley Orgánica de Comunicación; comunicación digital

\section{Social networks consumption in Ecuador An exploratory survey in Riobamba and Quito $(2013-14)$ \\ Abstract}

Digital communication in Ecuador is relatively differentiated particularly as a consequence of the low Internet connection rate in the Andean Republic.
Daniel Barredo Ibánez. Universidad de las Américas, Ecuador José María Pérez Zúniga. Universidad de Granada, España Montserrat Fernández. Universidad Central del Ecuador, Ecuador Rocío Vivas. Universidad Central del Ecuador, Ecuador Recibido el 28 de noviembre de 2014 Aceptado el 2 de diciembre de 2014 
According to official figures, in 2013 just four out of ten Ecuadorians had network connection. In addition, the communication context is in the middle of an interesting transformation process through the approval of instruments like the Communication Organic Law in 2013, which despite its exhaustive nature it does not regulate the communication contents issued by social network users. This article, through a survey targeted to citizens of two of the main cities of the country -Quito and Riobamba- intends to explore and determine how people living in these urban sites use social networks, and the possible age differences. The results indicate that YouTube, Facebook and Google are the social networks mostly used by Ecuadorians. In addition, after establishing that the major focus was on conventional social networks and iconic trends and those that allowed a mainly interpersonal use, the intergenerational and interprovincial gap was confirmed.

Keywords: Social networks; Ecuador; Communication Organic Law; digital communication

\section{Introducción: Ecuador, un país en transformación comunicacional}

La comunicación digital ecuatoriana se encuentra relativamente escalonada, debido particularmente a la baja tasa de conexión a Internet que hay en la República andina: solo cuatro de cada diez ecuatorianos, en 2013, tenían conexión a la red (Ministerio de Telecomunicaciones, 2014, 19 de mayo). El país presenta numerosas deficiencias que impiden un mayor número de usuarios de la ciberesfera:

"<...> hay otros factores que intervienen al margen de la falta de infraestructuras en algunas zonas del país, o la carestía de los equipos de conexión. Dentro de esas problemáticas, por ejemplo, destaca el hecho de que según cifras oficiales alrededor del 20,8\% de la población del Ecuador, en 2013, fuera clasificada

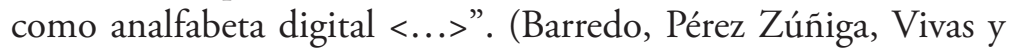
Fernández, 2015: en prensa)

Sin embargo, entre 2013 y 2006 hay que resaltar que el número de usuarios se ha multiplicado hasta por once (El Comercio.com, 2014, 17 de agosto). En términos globales, el país se encuentra en un 
proceso de repensar la comunicación, sobre todo tras la aprobación en 2013 de un aparataje legal tan innovador como el que presenta la Ley Orgánica de Comunicación [LOC], la cual responde, de una parte, a un interés regulatorio del gobierno de Rafael Correa, coincidente con el marco legislativo de la comunicación definido en muchos países latinoamericanos en los últimos tiempos (Ávila, 2013); y, de otra parte, a la crisis de credibilidad que atraviesan los medios de comunicación ecuatorianos y que ha sido descrita en algunos trabajos previos (Vásconez, 2011; Rodrigo, 2012; Barredo, Pérez Zúniga, Vivas y Fernández, 2015). Quizá la mayor crítica que recibe la LOC es la falta de aplicación de algunos de sus supuestos más importantes, como el polémico tema de la distribución de frecuencias; en el art. 106, la LOC establece que el espacio radioeléctrico debe repartirse en tres partes: "33\% de estas frecuencias para la operación de medios públicos, el 33\% para la operación de medios privados, y 34\% para la operación de medios comunitarios". A comienzos de 2015, sin embargo, casi dos años después de la aprobación del texto jurídico, Chavero y Oller (2015) explican que los medios comunitarios representan alrededor de un 3\% del país, por más del 90\% de los medios privados.

\section{El cambio de las actitudes a través de las redes sociales}

Las redes sociales escapan al ámbito regulatorio de la LOC (Barredo, Pérez Zúñiga, Vivas y Fernández, 2015), con lo cual, desde nuestro punto de vista, pueden erigirse como focos de insurgencia en un paradigma dominado por ese "modelo autoritario - competitivo del gobierno de Rafael Correa”, en palabras de Basabe-Serrano y Martínez (2014: 146). Sin ofrecer una crítica tan gruesa, autores como Ramos (2013: 76) mencionan el "activismo estatal" de los gobiernos de la Revolución Ciudadana, su capacidad para enfrentar un paradigma esencialmente dominado por el neoliberalismo, y su lucha constante contra los gobiernos progresistas latinoamericanos (Ramos, 2012). También es cierto que ese activismo se cimenta en un elevado control del Estado en el mercado publicitario (Checa - Godoy, 2012), y en un interés presidencial hasta cierto punto mesiánico que asegura que quiere "dejar un país que viva 
permanentemente en la verdad, combatiendo a esta prensa corrupta" (El

Comercio.com, 2011, 10 de diciembre). Asimismo, se han observado numerosos "actores gubernamentales" (Ramos y Gómez, 2014: 307) en esos organismos recientemente creados -como el Consejo de Regulación y Desarrollo de la Información y Comunicación [CORDICOM], o la Superintendencia de la Información y Comunicación [SUPERCOM]-, en detrimento de representantes procedentes de esferas civiles, profesionales $\mathrm{o}$ académicas.

Las redes sociales han tenido un enorme protagonismo en la esfera pública ecuatoriana; investigadores como León (2007) o Rivera Costales (2014), por ejemplo, les otorgan una gran capacidad de influencia en momentos electorales como la campaña de 2006, donde salió vencedor Rafael Correa. Desde ese punto de vista, la importancia es tal que algunas instituciones ecuatorianas están implementando plataformas digitales que permiten profundizar el sentido de la democracia, según describen Barredo y Silva (2014).

La mayor ventaja que ofrecen las redes sociales a los candidatos o líderes políticos y/u organizacionales es la posibilidad de diseñar campañas de bajo coste, y particularmente la facilidad de contactar directamente a los públicos estratégicos (Valdivieso, 2008).

Pero a pesar del cambio actitudinal anteriormente descrito, algunas de las instituciones ecuatorianas no impulsan estrategias activas para conectar con los usuarios de la ciberesfera (Barredo, 2014), quizá porque son incapaces de percibir el llamado "cambio de paradigma" (Barredo, Oller y Buenaventura, 2013: 79) promovido por las redes sociales. Esa transformación está anclada en una revisión del posicionamiento de los usuarios o consumidores de los medios, quienes han respondido al empuje creciente de las nuevas tecnologías mediante el desarrollo de las siguientes alteraciones:

a) Cambios actitudinales. Los consumidores de los medios de comunicación del siglo XXI demandan participar de la información, hasta el punto de que está emergiendo un nuevo contexto relacional (Barredo, Oller y Buenaventura, 2013), en donde el usuario demanda intervenir activamente en el ecosistema simbólico que lo acoge (Bruns, 2007). 
b) Culturales. La aparición del llamado ciberactivismo, como explica Caldevilla (2009), ha originado a un tipo de ciudadano que -independientemente de las ideologías- utiliza las TIC para promover una mayor profundización política. El impulso de los ciberactivistas ha conseguido extraordinarios logros, como el perdón del rey Juan Carlos en España en 2012 tras la llamada crisis de los elefantes (Barredo, 2013).

c) Informativos. Se está produciendo una emergencia de una actitud cada vez más informativa de los usuarios de los cibermedios; no nos referimos únicamente al periodismo ciudadano mencionado por Bowman y Willis (2003), sino sobre todo a la integración de los usuarios en formas periodísticas híbridas. Asimismo, el consumo se ha visto influenciado por la aparición de una "comunicación multidireccional" (Túnez y Sixto, 2011: 2), mediante la cual los usuarios tienden a demandar contenidos elaborados en distintos lenguajes y en diferentes plataformas.

Pero a pesar de la importancia de estas transformaciones, en el caso de Ecuador podría decirse que se ha investigado relativamente poco sobre la sociedad digital, y es por ello que nos hemos planteado la siguiente pregunta de investigación:

¿Qué redes sociales tendían a frecuentar los usuarios ecuatorianos de dos entornos urbanos simbólicamente representativos del país?

La respuesta a esta pregunta permitirá apuntalar algunas impresiones sobre un contexto que, junto al interés de sus reformas políticas recientes, se une el desconocimiento parcial sobre sus particularidades.

\section{Metodología}

El presente estudio, complementario a otro (Barredo, Pérez Zúńiga, Vivas y Fernández, 2015), ha trazado un enfoque exploratorio, mediante la aplicación de una encuesta (Ander-Egg, 1993). En total, diseñamos veinte interrogantes, con las que pretendíamos cuantificar 
aspectos vinculados a la contextualización, a los perfiles de uso y a las percepciones sobre la credibilidad de los medios que tenía un conjunto de encuestados. Debido a la falta de financiación del proyecto, decidimos ajustar la evaluación a dos capitales simbólicamente importantes de Ecuador:

"Quito, capital del país, radicada en Pichincha, donde se encuentra el mayor número de personas que empleaban computador y teléfonos celulares en 2013 (INEC, s.f.). Y Riobamba, capital de Chimborazo, la décima provincia del Ecuador en el uso de computadores y la última en el número de teléfonos celulares activados, según la misma fuente". (Barredo, Pérez Zúñiga, Vivas y Fernández, 2015: en prensa).

Los datos vinculados a Riobamba fueron generados en noviembre de 2013 gracias a la ayuda del Grupo de Alto Rendimiento de la Escuela Superior Politécnica de Chimborazo. La información sobre Quito, por su parte, se levantó entre marzo y junio de 2014 mediante el apoyo de varios grupos de estudiantes de la Facultad de Comunicación de la Universidad Central del Ecuador.

Se escogió un muestreo no probabilístico de cuotas, según el cual se pidió a los estudiantes de Riobamba y de Quito recoger "cierto número de entrevistas (cuotas)" (Pimienta Lastra, 2000: 266). Pero la inexperiencia de los encuestadores originó las deficiencias o descompensación en las cuotas que detectamos a la hora de analizar las matrices de datos; y hay que recordar que este tipo de muestreo, en cambio, impide "la revisita" (Núñez, 2005: 228) para solventar perjuicios como los aludidos. De todos modos, los resultados que vamos a mostrar a continuación tienen un carácter exploratorio, es decir, no deben generalizarse, si bien su interés radica en aportar ideas y sugerencias para un ámbito -el del consumo de las redes sociales en Ecuador- particularmente vacío de literatura. 
Globalmente, se consolidó una muestra formada por 1073 opiniones, de las cuales 547 procedían de Riobamba y 526 de Quito:

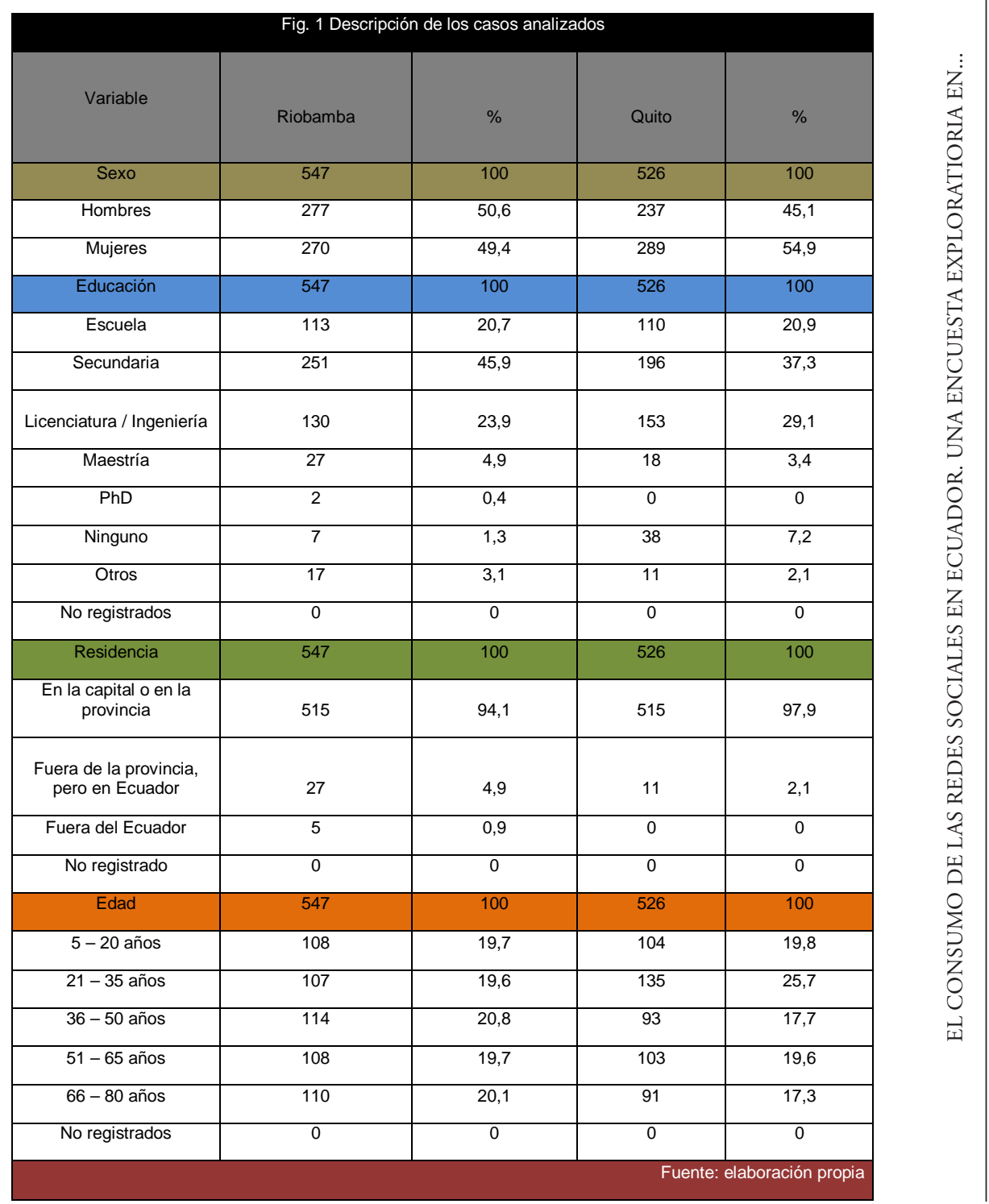


Las muestras obtenidas, según es posible observar en la figura 1, resultan en general equivalentes, a pesar de que algunos ítems registran una varianza de hasta un $9 \%$.

\section{Resultados}

A nivel macro, la opinión de los encuestados de una y otra capital, tendía a considerar a Facebook como la red social más frecuentada, unos resultados que coinciden con los obtenidos a través de otras metodologías (El Comercio.com, 2014, 17 de agosto):

Fig. 2 Redes sociales más frecuentadas en Riobamba y Quito (2013 - 14)

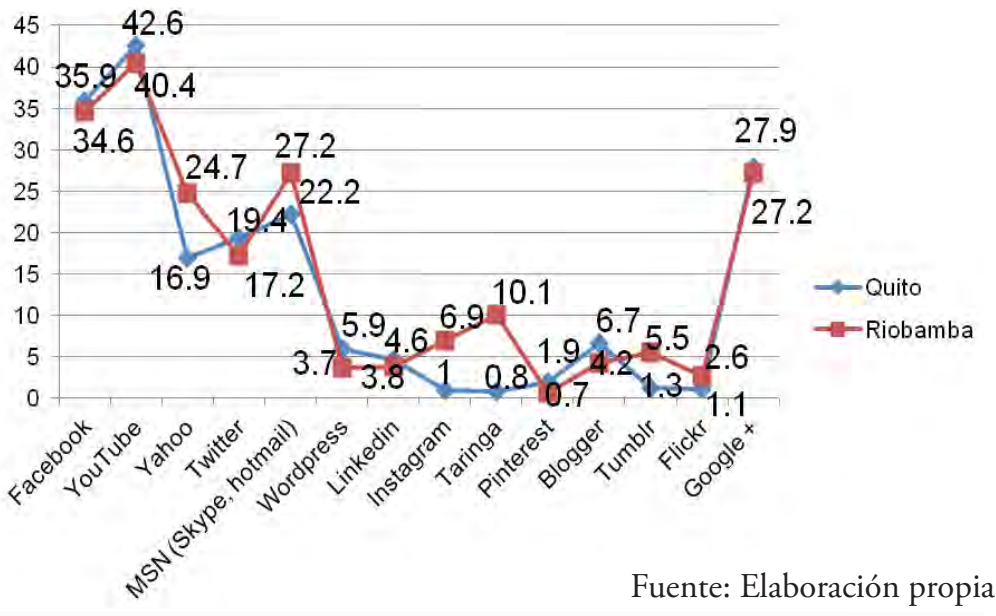

Algunas redes sociales importantes a nivel mundial, como por ejemplo Instagram (El Comercio.pe, 2014, 16 de diciembre), obtenían porcentajes marginales en comparación con las principales páginas visitadas, según confesaron los encuestados. Si se analiza atentamente el gráfico anterior, puede catalogarse el comportamiento de los usuarios ecuatorianos en función de las siguientes tendencias:

Tendencias icónicas. Determinadas por el consumo de redes donde imperaban, fundamentalmente, los vídeos o las fotografías, como YouTube y Facebook. 
Tendencias interpersonales. Porque los usuarios tendían a asignar una mayor importancia a aquellas redes que, como el paquete MSNo Google +, podían ser empleadas no específicamente para dirigirse a un colectivo, sino para establecer soluciones de comunicación interpersonal.

Tendencias vinculadas a la instantaneidad. Representadas sobre todo por la red Twitter, aparecieron en un tercer nivel, con casi la mitad de usuarios. Estas tendencias podían estar orientadas a usuarios altamente cualificados, pertenecientes a diversas élites ciudadanas.

Tendencias vinculadas al uso profesional. Los encuestados no manifestaron un uso extensivo de la red LinkedIn, tal vez debido a las bajas tasas de conexión a Internet que había en el Ecuador y, por consiguiente, la escasa apertura de las organizaciones o de los usuarios para promover una contratación activa mediante este servicio.

Tendencias reflexivas. Resultaron ser marginales, particularmente por los escasos niveles de uso de canales como las bitácoras.

Por otra parte, percibimos un uso etario de las redes sociales, el cual estaba relacionado con el perfil cultural de las personas encuestadas, así como con otros aspectos -como la longevidad de la red social o la tendencia social en el ámbito urbano:

Fig. 3 Percepción importante o muy importante de los encuestados sobre las

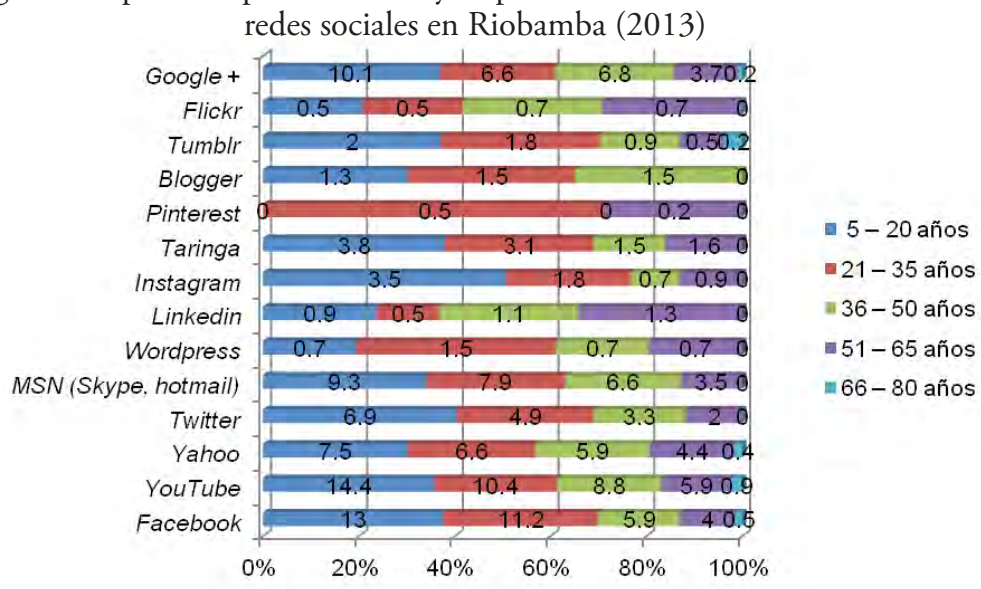

Fuente: Elaboración propia 
Fig. 4 Percepción importante o muy importante de los encuestados sobre las redes sociales en Quito (2014)

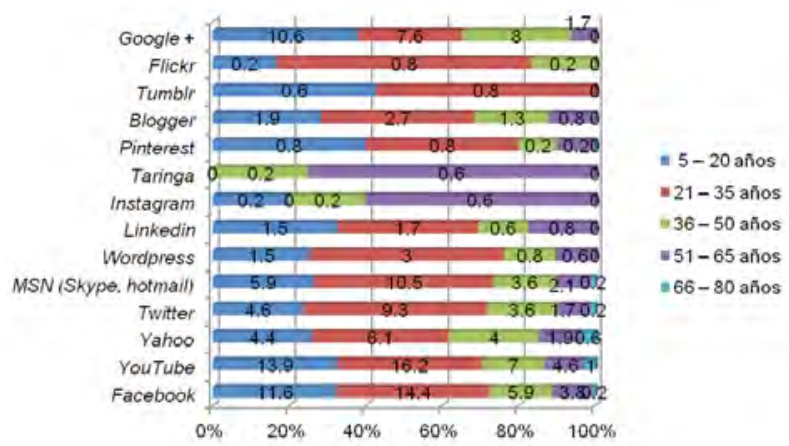

Fuente: Elaboración propia

Las personas de avanzada edad (de entre 66 y 80 años) presentaban un menor volumen de conexión global a las redes y, además, tendían a concentrarse fundamentalmente en las redes sociales más populares. El grupo etario más significativo según su presencia en redes, era el de los encuestados que oscilaban entre los 21 y los 35 años.

Asimismo, anotamos algunas diferencias en el comportamiento de los usuarios de uno y otro entorno, como por ejemplo en el caso de la red de uso profesional -LinkedIn-; en Riobamba, el grupo dominante oscilaba entre los 36 y los 65 años, mientras que en Quito había un grupo de 35 ańos o menos que solían emplear más a menudo este canal. En la mayor parte de los ítems, con todo, había cierta variación entre los dos grupos, señal de que las metodologías de carácter extensivo no resultan prácticas para cuantificar el comportamiento o las tendencias de los usuarios.

\section{Conclusiones}

Erróneamente, en ocasiones, se explica que el uso de una red social determinada conduce a generar un mayor impacto, como por ejemplo relacionaba Mathew Carpenter al describir la utilización de Twitter por parte de los candidatos ecuatorianos a las elecciones municipales de 2014 (El Telégrafo, 2014, 28 de julio). No todas las redes sociales 
promueven el mismo impacto, sino que hay características contextuales que determinan la elección de unas $\mathrm{u}$ otras para ampliar la audiencia potencial en el caso de una campaña electoral; en ese sentido, aquellas organizaciones que estén interesadas en desarrollar campańas que reproduzcan eficazmente sus contenidos, deberían elaborar estudios como el presente para intentar identificar las particularidades locales y nacionales. Recordemos que Instagram, por ejemplo, a nivel global ha superado en volumen de usuarios a Twitter (El Comercio.pe, 2014, 16 de diciembre), si bien en Ecuador, según los resultados aportados anteriormente, la red social vinculada a la fotografía ha obtenido porcentajes muy bajos de utilización.

La considerable brecha digital del Ecuador, avisada por trabajos previos (Torres e Infante, 2011), se ha visibilizado en las páginas anteriores en las abruptas preferencias de los usuarios; por decirlo de otra manera, la atención se concentraba sobre todo en las redes sociales convencionales y en las tendencias icónicas y en esas otras que permitían un uso esencialmente interpersonal. La falta de participación de los ecuatorianos en los medios convencionales (Ayala y Calvache, 2009) se prolongaba también en los cibermedios, al determinar unas rutinas de participación fuertemente polarizadas. En términos de impacto, hay un porcentaje importante de usuarios que mantienen una opción indecisa, por ejemplo, en las rutinas electorales (Barredo, Rivera y Amézquita, 2015), y es por ello que los gestores organizacionales deberían entonces emplear redes focalizadas en élites sociales -como Twitter-, sin descuidar la actividad en redes que permiten contactar a esos grupos que concentran su actividad en redes icónicas -como YouTube y Facebook.

En trabajos posteriores, queda pendiente realizar análisis cualitativos que complementen a las páginas precedentes y que ayuden a explicar los cambios actitudinales, culturales e informativos, entre otros, que mencionábamos anteriormente.

\section{$\underline{\text { Referencias }}$}

Ander-Egg, Ezequiel (1993). Técnicas de Investigación Social. 23a edición. Buenos Aires: Magisterio del Río de La Plata. 
Ávila, Caroline (2013). El sistema de comunicación en la Ley Orgánica de Comunicación del Ecuador: Un análisis desde el enfoque de las teorías de sociedad y masas de McQuail. Chasqui, Revista Latinoamericana de Comunicación, 124, 71 - 79.

Ayala, Alexandra y Calvache, María Belén (2009). Percepción sobre los medios públicos en Ecuador. Quito: CIESPAL.

Barredo Ibáńez, Daniel (2013). El tabú Real. La imagen de una monarquía en crisis. Córdoba: Berenice.

Barredo, Daniel; Oller, Martín y Buenaventura, Sandra (2013). The End of the Information Society. Notes for the Configuration of the New Contemporary Public Space: the Society of Devisers. Observatorio (OBS*) Journal, 7(3), 079 - 091.

Barredo, Daniel (2014). El Modelo Integrado de Evaluación de la Comunicación Organizacional en Ecuador: un estudio exploratorio a partir del diagnóstico de la ESPOCH y de CIESPAL (2013-2014). index.communicación, 1(4), 173 - 208.

Barredo, Daniel y Silva, Roxana (2014). Interacción institucional, participación democrática y transparencia: la iniciativa del Voto Transparente en el Ecuador (2013 - 14). Chasqui, Revista Latinoamericana de Comunicación, 126, 4 - 13.

Barredo, Daniel; Rivera, José y Amézquita, Álex (2015). La influencia de las redes sociales en la intención de voto. Una encuesta a partir de las elecciones municipales en Ecuador (2014). Quórum Académico, 12(1).

Barredo, Daniel; Pérez Zúñiga, José María; Vivas, Rocío y Fernández, Montserrat (2015). "El periodismo ecuatoriano en entredicho. Descenso de credibilidad y nuevas perspectivas comunicacionales". En Barredo Ibáńez, Daniel; Oller, Martín y Hernández, Sergio (Eds.). La Comunicación y el Periodismo de Ecuador frente a los desafios contemporáneos. La Laguna: Latina.

Basabe-Serrano, Santiago y Martínez, Julián (2014). Ecuador: cada vez menos democracia, cada vez más autoritarismo... con elecciones. Revista de Ciencia Política, 34(1). 145 - 170.

Bowman, Shayne y Willis, Chris (2003). We media: how audiences are shaping the future of news and information. Reston: The Media Center at the American Press Institute. Recuperado el 14 de enero de 2015 de: http://www.hypergene.net/wemedia/download/we media.pdf 
Bruns, Axel (2007). Produsage: Towards a Broader Framework for User - Led Content Creation. En Proceedings Creativity \& Cognition, 6. Consultado el 16/01/2015 de: http://eprints.qut.edu. au/6623/1/6623.pdf Caldevilla, David (2009). Democracia 2.0: La política se introduce en las redes sociales. Pensar la Publicidad, III (2), $31-48$.

Chavero, Palmira y Oller, Martín (2015). "Politicas públicas en comunicación y sistemas mediáticos. El caso de Ecuador". En Barredo Ibáńez, Daniel; Oller, Martín y Hernández, Sergio (Eds.). La Comunicación y el Periodismo de Ecuador frente a los desafios contemporáneos. La Laguna: Latina.

Checa - Godoy, Antonio (2012). La Banca y la propiedad de los medios: el caso de Ecuador. Revista Latina de Comunicación Social, 67, 125 - 147. Consultado el 01/09/2014 de: http://www.revistalatinacs. org/067/art/950 Sevilla/06 Checa.html

El Comercio.com (2011, 10 de diciembre). Resumen del enlace sabatino. Consultado el 28/08/2014 de: http://www.elcomercio. com/actualidad/politica/resumen-del-enlace-sabatino.html

ElComercio.com (2014, 17 de agosto). El uso de Internet en Ecuador creció 11 veces en siete años. Consultado el 16/01/2015 de: http://www. elcomercio.com/tendencias/ecuador-internet-datos-tecnologiausuarios.html

ElComercio.pe (2014, 16 de diciembre). Director de Twitter se burla de número de usuarios de Instagram. Consultado el 16/01/2015 de: http://elcomercio.pe/redes-sociales/twitter/twitter-me-importa-mieque-instagram-tenga-mas-usuarios-noticia-1778176

El Telégrafo (2014, 28 de julio). "El Estado es el principal cliente de Ecuador en Twitter". Consultado el 16/01/2014 de: http://www. eltelegrafo.com.ec/economia/masqmenos/item/el-estado-es-elprincipal-cliente-de-ecuador-en-twitter.html

León, Osvaldo (2007). Ecuador: Elecciones, medios y democracia. Chasqui, Revista Latinoamericana de Comunicación, 97, 50 - 55.

Ministerio de Telecomunicaciones (2014, 19 de mayo). El 40,4\% de los Ecuatorianos utilizó internet en los últimos 12 meses. Recuperado el 1 de diciembre de 2014 de http://www.telecomunicaciones.gob.ec/el404-de-los-ecuatorianos-utilizo-internet-en-los-ultimos-12-meses/ 
Núńez, Adoración (2005). Incidencias de la entrevista personal en la investigación mediante encuesta. Revista Española de Investigaciones Sociológicas, 109, 219 - 236.

Pimienta Lastra, Rodrigo (2000). Encuestas probabilísticas vs. no probabilísticas. Política y Cultura, 13, 263 - 276.

Ramos, Isabel (2012). La contienda política entre los medios privados y el gobierno de Rafael Correa. Utopía y Praxis Latinoamericana, 17(58), $65-76$.

Ramos, Isabel (2013). Trayectorias de democratización y desdemocratización de la comunicación en Ecuador. Íconos. Revista de Ciencias Sociales, 45, $67-82$.

Ramos, Juan y Gómez, Ava (2014). Sujetos, objetos, decisiones y evasiones - el proceso de aprobación de la Ley de Comunicación en Ecuador. Intercom, Revista Brasileira de Ciências da Comunicação, 37(1), $283-310$.

Rivera Costales, José (2014). Rafael Correa y las elecciones 2006. Inicios del Marketing y Comunicación politica digital en Ecuador. Chasqui, Revista Latinoamericana de Comunicación, 126, 117 - 123.

Rodrigo, Iván (2012). Calidad informativa: credibilidad de medios y periodistas en el Ecuador. ComHumanitas, 3(3), 53 - 69.

Torres, Juan Carlos e Infante, Alfonso (2011). Desigualdad digital en la universidad: usos de Internet en Ecuador. Comunicar, 37(XIX), $81-88$.

Túnez, Miguel y Sixto, José (2011). Redes sociales, politica y Compromiso 2.0: La comunicación de los diputados españoles en Facebook. Revista Latina de Comunicación Social, 66, 1 - 25. Consultado el 12/03/2014 de: http://www.ull.es/publicaciones/latina/11/art/930_Santiago/ RLCS_art931.pdf

Valdivieso, Jeanneth (2008). "La confrontación que da éxito". En Rincón, Omar (Ed.). Los tele-presidentes: cerca del pueblo, lejos de la democracia (crónicas de 12 presidentes latinoamericanos y sus modos de comunicar) <pp. 75 - 83>. Bogotá: FES - C3.

Vásconez, Irene (2011). La credibilidad de los medios de comunicación en el Ecuador: reflejo de un periodismo de calidad. ComHumanitas, 1(1), $173-182$. 